\title{
Efficacy and safety of minimally invasive surfactant administration in preterm infants with neonatal respiratory distress syndrome: a systematic review and meta-analysis
}

\author{
Xiao Hong $\mathrm{Wu}^{1}$, Zhoushan Feng ${ }^{2}$, Juan Kong ${ }^{1}$, Yiyu Lai ${ }^{1}$, Chunhong Jia ${ }^{1}$, Zhanyuan Xu${ }^{1}$, \\ Fan $\mathrm{Wu}^{1}$, Qiliang $\mathrm{Cui}^{1}$, and Yaoyong $\mathrm{Chen}^{1}$ \\ ${ }^{1}$ Guangzhou Medical University \\ ${ }^{2}$ Sun Yat-sen University Sixth Affiliated Hospital
}

February 11, 2021

\begin{abstract}
Abstract Background: The effects of minimally invasive surfactant administration (MISA) in preterm infants with neonatal respiratory distress syndrome (NRDS) are unclear. Methods: We searched randomized controlled trials (RCTs) and compared MISA techniques with intubation for surfactant delivery in preterm infants with NRDS in PubMed, Embase, Cochrane Library, and Web of Science. Results: Thirteen RCTs (1931 infants) were included in the meta-analysis. The use of MISA techniques decrease the incidence of bronchopulmonary dysplasia (BPD) at 36 weeks, pneumothorax, and hemodynamically significant patent ductus arteriosus (hsPDA) (Risk Ratio(RR) : 0.59, 95\% confidence interval (CI) : 0.46 to 0.75, p <.0001; RR : 0.60, $95 \%$ CI : 0.39 to $0.93, \mathrm{p}=.02$ and $\mathrm{RR}: 0.88,95 \% \mathrm{CI}: 0.78$ to $1.00, \mathrm{p}=.04$, respectively). In addition, infants in the MISA group required less mechanical ventilation within $72 \mathrm{~h}$ of life or during hospitalization (RR : $0.60,95 \%$ CI : 0.48 to $0.75, \mathrm{p}<$ .00001 and $\mathrm{RR}: 0.64,95 \% \mathrm{CI}: 0.49$ to $0.82, \mathrm{p}=.0005$, respectively) compared with infants in the control group. However, the rate of surfactant reflux was higher in the MISA group than that in the control group (RR : $2.12,95 \%$ CI : 1.37 to $3.29, \mathrm{p}=$ .0008). There were no significant differences in mortality and other outcomes beteween the MISA group and the control group. Conclusions: The administration of surfactant with MISA techniques could lower the requirement for mechanical ventilation, and decrease the incidence of BPD at 36 weeks, pneumothorax, and hsPDA.
\end{abstract}

Efficacy and safety of minimally invasive surfactant administration in preterm infants with neonatal respiratory distress syndrome: a systematic review and meta-analysis

Xiaohong Wu MD ${ }^{1} \mid$ Zhoushan Feng $\mathrm{MD}^{2} \mid$ Juan Kong MD ${ }^{1}$ Yiyu Lai MD ${ }^{1}$ Chunhong Jia MD ${ }^{1} \mid$ Zhanyuan $\mathrm{Xu} \mathrm{MD}^{1} \mid$ Fan Wu MD ${ }^{1}$ |Qiliang Cui $\mathrm{MD}^{1} \mid$ Yaoyong Chen $\mathrm{MD}^{1}$

${ }^{1}$ Department of Pediatrics, The Third Affifiliated Hospital of Guangzhou Medical University, Guangzhou, China

${ }^{2}$ Department of Pediatrics, The Sixth Affifiliated Hospital of Sun Yat-sen University, Guangzhou, China

Xiaohong Wu and Zhoushan Feng share first authorship

\section{Correspondence}

Yaoyong Chen MD, QiLiang Cui MD and Fan Wu MD,

Department of Pediatrics

The Third Affifiliated Hospital of Guangzhou Medical University 
Guangzhou, Guangdong 510140, China

E-mail:

ychen516@163.com,

1551838354@qq.com and

gdwufan@126.com

\section{Abstract}

Background:The effects of minimally invasive surfactant administration (MISA) in preterm infants with neonatal respiratory distress syndrome (NRDS) are unclear.

Methods: We searched randomized controlled trials (RCTs) and compared MISA techniques with intubation for surfactant delivery in preterm infants with NRDS in PubMed, Embase, Cochrane Library, and Web of Science.

Results: Thirteen RCTs (1931 infants) were included in the meta-analysis. The use of MISA techniques decrease the incidence of bronchopulmonary dysplasia (BPD) at 36 weeks, pneumothorax, and hemodynamically significant patent ductus arteriosus (hsPDA) (Risk Ratio(RR) : 0.59, 95\% confidence interval (CI) : 0.46 to $0.75, \mathrm{p}<.0001 ; \mathrm{RR}: 0.60,95 \% \mathrm{CI}: 0.39$ to $0.93, \mathrm{p}=.02$ and $\mathrm{RR}: 0.88,95 \%$ CI $: 0.78$ to 1.00 , $\mathrm{p}=.04$, respectively). In addition, infants in the MISA group required less mechanical ventilation within $72 \mathrm{~h}$ of life or during hospitalization (RR : $0.60,95 \%$ CI $: 0.48$ to $0.75, \mathrm{p}<.00001$ and $\mathrm{RR}: 0.64,95 \%$ CI : 0.49 to $0.82, \mathrm{p}=.0005$, respectively) compared with infants in the control group. However, the rate of surfactant reflux was higher in the MISA group than that in the control group (RR : $2.12,95 \% \mathrm{CI}: 1.37$ to $3.29, \mathrm{p}=.0008)$. There were no significant differences in mortality and other outcomes beteween the MISA group and the control group.

Conclusions: The administration of surfactant with MISA techniques could lower the requirement for mechanical ventilation, and decrease the incidence of BPD at 36 weeks, pneumothorax, and hsPDA.

\section{KEYWORDS}

bronchopulmonary dysplasia; neonatal respiratory distress syndrome; minimally invasive surfactant administration; intubation-surfactant-extubation strategy; meta analysis1 | INTRODUCTION

Neonatal respiratory distress syndrome (NRDS), one of the most common respiratory diseases in preterm infants, is mainly due to the lack of pulmonary surfactant, which often results in neonatal death ${ }^{1}$. Exogenous pulmonary surfactant replacement therapy can reduce the incidence of NRDS and neonatal mortality and improve the long-term outcome; therefore, it is currently recognized as the most effective treatment for NRDS ${ }^{2-4}$. Previous treatment options for NRDS included mechanical ventilation with surfactant replacement therapy, except that this approach resulted in a high incidence of ventilator-associated lung injury and bronchopulmonary dysplasia (BPD) ${ }^{5,6}$. Intubation, surfactant administration, and extubation (INSURE) techniques were later developed, which could effectively reduce the incidence of BPD and the duration of mechanical ventilation ${ }^{7,8}$. However, endotracheal intubation can damage the airway ${ }^{9}$, and transient positive pressure ventilation can cause significant lung injury ${ }^{10}$.

In order to prevent intubation for surfactant delivery in preterm infants with NRDS, MISA techniques are recommended, including less invasive surfactant administration (LISA) and minimally invasive surfactant therapy (MIST) ${ }^{11}$. These techniques are characterized with the use of a thin catheter for surfactant delivery while the infant breathes spontaneously with the support of nasal continuous positive airway pressure (nCPAP). Nevertheless, it is still unknown whether MISA techniques can reduce the mortality rate, as well as the incidence of BPD and other neonatal complications compared with conventional intubation techniques. Therefore, we systematically searched randomized controlled trials (RCTs), compared MISA techniques with endotracheal intubation for surfactant delivery, and conducted meta-analysis to further clarify their efficacy and safety. 


\section{2 | METHODS}

This systematic review was conducted in accordance with the Preferred Reporting Items for Systematic Reviews and Meta-analyses (PRISMA) guidelines ${ }^{12}$.

\section{1 | Search strategy}

Searches were conducted in PubMed, Embase, Cochrane Library, and Web of Science databases from inception to November 4, 2020. Keywords and MeSH terms were as follows: "Infant, Premature", "Infant, Extremely Premature", "Premature Birth", "MISA", "MIST", "LISA", "less invasive", "minimally invasive", "thin catheter", "gastric tube", "nasogastric tube", "non-intubated", "non-intubation", "noninvasive", "lung surfactant", "pulmonary surfactant", and "surfactant". We also reviewed the references in each included study to identify any relevant papers that might have been missed from the search.

\section{2 | Inclusion criteria}

(1) Participants: premature infants diagnosed with NRDS (gestational age, $<37$ weeks).

(2) Research type: published clinical RCTs written in English.

(3) Intervention measures: the intervention group applied MISA techniques for surfactant delivery, whereas the control group applied endotracheal instillation.

(4) One or more of the following outcomes was measured in the literature: the mortality rate, the requirement for mechanical ventilation, or the presence of any other neonatal morbidity.

\section{3 | Study selection and data extraction}

After Endnote software was used to remove duplicate studies, two reviewers (Xiaohong Wu and Zhoushan Feng) independently selected eligible studies and extracted data according to the inclusion criteria. Differences were settled by discussion and consensus. For studies with incomplete information, if the missing data could not be obtained after contacting the author, a group discussion was conducted to decide whether the study should be included.

\section{4 | Study quality assessment}

Two reviewers (Xiaohong Wu and Zhoushan Feng) independently evaluated data in randomized studies using the Cochrane "Risk of Bias Assessment Tool" 13. Parameters for evaluation were as follows: adequacy of random sequence generation, allocation concealment, blinding of the caregivers and the assessors, incomplete outcome data reporting, and selective reporting. Discrepancies were settled by discussion and consensus.

\section{5 | Data analysis}

Meta-analysis was performed by Review Manager 5.3 (The Cochrane Collaboration, Copenhagen, Denmark) and Stata 14.0 (Stata Corporation, College Station, TX, USA). Effect sizes computed for the analyses were presented as risk ratios (RR) with $95 \%$ confidence intervals (CI). The RR was not estimable when the events were zero in both groups. $\mathrm{I}^{2}<50 \%$ was indicative of no heterogeneity, and the fixed effect model was adopted. By contrast, $\mathrm{I}^{2}>50 \%$ was indicative of heterogeneity, and the random effect model was applied after sensitivity analysis. Publication bias was assessed visually by funnel plots and then by Egger tests. P $<.05$ was considered statistically significant.

\section{3 | RESULTS}

\subsection{Study selection and characteristics of included studies}

A total of 915 studies were obtained during the initial search. Subsequently, 371 duplicates were distinguished and excluded with Endnote software. From the remaining 544 articles, 496 articles were exempted after scanning titles and abstracts. Finally, 35 articles were excluded after reading the full text, and 13 articles ${ }^{14-26}$ were included in the meta-analysis with a total of 1931 infants, including 964 in the MISA group and 967 in the control group. 
The control groups in two articles ${ }^{14,19}$ chose mechanical ventilation after intubation for surfactant delivery, whereas the intervention groups in 11 articles ${ }^{15-18,20-26}$ chose INSURE techniques. The MISA techniques used in the intervention groups were as follows: eight articles ${ }^{14,16-20,22,26}$ used LISA techniques, two articles ${ }^{21,24}$ used MIST techniques, two articles ${ }^{23,25}$ used both techniques, and one article ${ }^{15}$ only mentioned administration of a surfactant via a thin catheter but did not specify which technique was used.

The application of cesarean sections and antenatal steroids was similar between the two groups in each study. All studies described similar criteria for surfactant delivery with similar values of fractional oxygen inhalation $\left(\mathrm{FiO}_{2}\right)$ and/or respiratory distress severity, whereas 12 articles ${ }^{14,16-26}$ described criteria for rescue intubation. In ten articles ${ }^{14-21,24,26}$, the type of pulmonary surfactant was Curosurf, whereas in three articles ${ }^{22,23,25}$, three other kinds of pulmonary surfactant were used. The literature screening process is shown in Figure 1, and the basic characteristics of the included studies are given in Table 1.

\subsection{Risk of bias assessment for all included studies}

All included studies were generally considered to be low or moderate risk according to the Cochrane assessment standards for RCT. Among them, 11 trials ${ }^{14,16,18-26}$ reported the method of random sequence generation, and 11 trials ${ }^{14,16,19-26}$ described the specific allocation concealment methods. Due to the particularity of the intervention, blinding was not possible in all trials, and blinding of researchers and subjects was assessed as high risk. However, almost all studies described the criteria for surfactant delivery ${ }^{14-26}$ and rescue intubation ${ }^{14,16-26}$, so as to minimize such bias. Twelve articles ${ }^{14,16-26}$ reported complete outcome data. For reporting bias, five studies ${ }^{15,17,18,22,23,26}$ showed unclear bias because they were not registered in a public trial registry, and three studies ${ }^{20,24,25}$ showed high risk (Table 2).

\section{3 | Outcomes}

\subsection{1 | All-cause mortality}

Mortality data were reported in 11 studies ${ }^{14-23,26}$, in which one study ${ }^{26}$ had a mortality rate of 0 , so it was not included in the meta-analysis. There was no significant difference in the mortality rate between the MISA group and the control group (Risk ratio (RR) : 0.75, 95\% confidence interval (CI) : 0.56 to $1.00, \mathrm{I}^{2}=$ $8 \%, \mathrm{p}=.05,10$ studies $\left.^{14-23}(\mathrm{n}=1478)\right)$.

As the subjects in two studies ${ }^{14,19}$ were preterm infants at $<29$ weeks and for the control group, instead of choosing INSURE techniques for surfactant delivery, the authors selected a more traditional approach, that is, continuous mechanical ventilation after surfactant instillation with endotracheal intubation, sensitivity analysis yielded different results ( $\mathrm{RR}: 0.69,95 \% \mathrm{CI}: 0.51$ to $0.95, \mathrm{p}=.02, \mathrm{I}^{2}=17 \%, 8$ studies $^{15-18,20-23}$ (n $=1047)$ ) (Figure 2A).

\subsection{2 $\mid \mathrm{BPD}$ at 36 weeks}

Twelve trials ${ }^{14-21,23-26}$ involving 1831 newborns reported the incidence of BPD at 36 weeks. Meta-analysis revealed that MISA techniques brought about a significant decrease in this outcome (RR : 0.59, 95\% CI : 0.46 to $\left.0.75, \mathrm{I}^{2}=3 \%, \mathrm{p}<.0001\right)$. Sensitivity analysis limited to studies that unequivocally stated the use of the INSURE regimen in the control group showed similar results (RR : $0.59,95 \%$ CI : 0.45 to $0.78, \mathrm{I}^{2}=$ $20 \%, \mathrm{p}=.0002,10$ studies $^{15-18,20,21,23-26}(\mathrm{n}=1400)$ ) (Figure $\left.2 \mathrm{~B}\right)$.

\subsection{3 | Need for mechanical ventilation within $72 \mathrm{~h}$ of life}

Eight trials ${ }^{14,16-18,20,21,24,26}$ showed data on the requirement of mechanical ventilation within $72 \mathrm{~h}$ of life with a total of 892 infants. Meta-analysis revealed that infants in the MISA group required less mechanical ventilation within $72 \mathrm{~h}$ of life (RR : $0.60,95 \% \mathrm{CI}: 0.48$ to $\left.0.75, \mathrm{I}^{2}=36 \%, \mathrm{p}<.00001\right)$. Sensitivity analysis showed similar results (RR : $0.76,95 \%$ CI : 0.58 to $0.99, \mathrm{I}^{2}=0 \%, \mathrm{p}=.04,7$ studies $^{16-18,20,21,24,26}(\mathrm{n}=672)$ ) (Figure 2C).

\subsection{4 | Need for mechanical ventilation during hospitalization}


In this meta-analysis, the random effect model (REM) was applied, as significant heterogeneity was observed in the fixed-effect model (FEM) $\left(\mathrm{I}^{2}=72 \%\right)$. Meta-analysis estimated that the MISA group had a lesser need for mechanical ventilation during hospitalization (RR : $0.64,95 \%$ CI : 0.49 to $0.82, p=.0005,8$ studies $\left.^{14-17,19,21-23}(\mathrm{n}=1350)\right)$. The heterogeneity did not change significantly after removing each trial one by one. The exclusion of two studies ${ }^{14,19}$ yielded similar results and failed to significantly reduce the heterogeneity (RR : $0.66,95 \% \mathrm{CI}: 0.48$ to $0.91, \mathrm{I}^{2}=51 \%, \mathrm{p}=.01,6$ studies $^{15-17,21-23}(\mathrm{n}=919)$ ) (Figure $2 \mathrm{D})$.

\subsection{5 | Other outcome measures}

Meta-analysis revealed a significantly lower incidence of pneumothorax and hsPDA in the MISA group than that in the control group (RR : $0.60,95 \%$ CI : 0.39 to 0.93$], \mathrm{p}=.02,9$ studies $^{14-19,21,22,26}(\mathrm{n}=1187)$ and $\mathrm{RR}$ : $0.88,95 \%$ CI $: 0.78$ to $1.00, \mathrm{p}=.04,11$ studies ${ }^{14-19,21-25}(\mathrm{n}=1796)$, respectively). There were no significant differences between the two groups in the other neonatal complications, namely, pulmonary hemorrhage, retinopathy of prematurity (ROP) (>stage II or the need for treatment), intraventricular haemorrhage (IVH) (all grades), severe IVH (grade III or higher), and periventricular leukomalacia (PVL). For procedurerelated outcomes, meta-analysis showed that MISA techniques increased the rate of surfactant reflux (RR $: 2.12,95 \%$ CI $: 1.37$ to $3.29, \mathrm{p}=.0008,5$ trials $^{16-18,21,26}(\mathrm{n}=576)$ ), but did not increase the need for additional surfactant compared with the control group (RR : 1.17, 95\% CI : 0.95 to $1.45, \mathrm{p}=.15,11$ trials $\left.{ }^{15-22,24-26}(\mathrm{n}=1658)\right)$. Sensitivity analysis was performed, and the heterogeneity of each outcome did not change significantly (Table 3 ).

\section{4 $\mid$ Publication bias}

Publication bias in the literature reporting the mortality rate was assessed by constructing a funnel plots and performing the Egger test. The funnel plot was symmetrical visually and verified by the Egger test (p $=.896$ ) (Figure 3).

\section{4 | DISCUSSION}

In this study, a systematic review of 13 RCTs demonstrated that the use of MISA techniques could significantly reduce the incidence of BPD at 36 weeks, pneumothorax, and hsPDA without increasing the incidence of other neonatal complications compared with endotracheal intubation for surfactant delivery. MISA techniques also reduced the need for mechanical ventilation within $72 \mathrm{~h}$ of life or during hospitalization. Although MISA techniques increased the rate of surfactant reflux, they did not increase the need for additional surfactant, indicating that MISA techniques did not reduce the efficiency of surfactant.

In addition, there was no significant difference in the mortality rate between the two groups. On the one hand, the mortality rate was lower in the MISA group than that in the control group $(9.1 \%$ vs. $12.1 \%)$, and it was right on the edge of statistical significance $(\mathrm{p}=.05)$, so further analysis is needed after additional RCT studies are published. On the other hand, the mortality rate was significantly reduced in the MISA group after sensitivity analysis $(\mathrm{RR}=0.69,95 \% \mathrm{CI}: 0.51$ to $0.95, \mathrm{p}=.02)$. This reduction might have been due to the younger gestational age of subjects in the excluded study (gestational age, $<29$ weeks) 14,19 . The younger the gestational age, the higher the mortality rate ${ }^{27,28}$. The effect of gestational age on mortality was greater than that of surfactant delivery techniques between the two groups. Overall, the results concluded here were robust to most sensitivity analyses.

Pneumothorax occurred less frequently in the MISA group than in the control group, considering that MISA techniques do not cause pressure or volume damage from brief mechanical ventilation. Similarly, the incidence of hsPDA in the MISA group was lower than that in the control group, suggesting that MISA techniques had fewer effects on hemodynamics. Epidemiological studies have reported that hsPDA associates with BPD ${ }^{29}$. hsPDA increased the pressure of pulmonary capillary bed arteries and veins, resulting in more fluid infiltration into the pulmonary interstitium, which aggravated pulmonary edema. In this study, the incidence of hsPDA in the MISA group was lower than that in the control group, which might have been another reason for the low incidence of BPD. 
The use of INSURE techniques for surfactant delivery has been widely reported in clinical practice, but many shortcomings remain ${ }^{30,31}$. Surfactant delivery in a noninvasive approach that reduces the hazards of endotracheal intubation, and it includes aerosolized administration ${ }^{32}$, laryngeal mask airway-guided administration ${ }^{33,34}$, pharyngeal administration ${ }^{35}$, and minimally invasive pulmonary surfactant administration. However, due to the lack of relevant studies on the first three approaches, the results are inconsistent, and further studies are needed to evaluate their safety and effectiveness ${ }^{36,37}$. Compared with endotracheal intubation, the possible advantages of minimally invasive administration are as follows. Firstly, thin tubes can reduce damage to vocal cords and the tracheal mucosa ${ }^{9}$. Secondly, MISA techniques can prevent lung injury in premature infants from high pressure or lung overdistension ${ }^{10,38}$. Finally, they allow uninterrupted application of NCPAP during surgery, thereby facilitating the maintenance of stable oxygen saturation ${ }^{39}$.

This meta-analysis searched for RCT studies using a thin catheter for surfactant delivery and included LISA and MIST techniques, leading to differences from other meta-analyses. A previously published metanarrative review failed to identify a lesser BPD at 36 weeks in the MISA group ${ }^{40}$, but this study observed a reduction in BPD at 36 weeks, which was consistent with the conclusions of a mesh meta-analysis and a cross-sectional observational multicenter study ${ }^{41,42}$. In comparison with a recent meta-analysis ${ }^{43}$, after excluding the same two RCTs for sensitivity analysis, this study found a reduction in mortality, whereas the authors of the previously published meta-analysis did not.

Although we strived for a comprehensive and accurate meta-analysis, there were several limitations. First, there were differences in the gestational age and the body weight among the studies, and lung tissues at different stages of development showed different sensitivities to injury from mechanical ventilation, which might have affected the incidence of BPD at 36 weeks, pneumothorax, and other complications. Second, different doses and types of surfactant might have affected the outcome of the study. Due to the limited number of existing studies, it was difficult to evaluate the aforementioned factors in the groups, and additional large-sample, multi-center clinical studies are needed to further explore the efficacy and safety. Finally, none of the included studies reported long-term neurodevelopmental follow-up data.

In conclusion, the use of MISA techniques for surfactant delivery not only reduced the need for mechanical ventilation (within $72 \mathrm{~h}$ of life or during hospitalization), but also reduced the incidence of BPD at 36 weeks, pneumothorax, and hsPDA without increasing the incidence of other complications. Therefore, MISA techniques should be considered as part of the lung protective strategy for premature infants with NRDS. However, the specifics of these techniques are rarely reported and related large-sample multicenter studies are scarce. Therefore, additional well-designed, well-targeted RCT studies are needed to evaluate their efficacy and safety.

\section{A CKNOWLEDGEMENT}

We thank International Science Editing ( http://www.internationalscienceediting.com ) for editing this manuscript.

\section{CONFLICT OF INTERESTS}

The authors have no conflicts of interest to declare.

\section{AUTHOR CONTRIBUTIONS}

Xiaohong Wu and Zhoushan Feng conceptualized and designed research, collected and analyzed data, and drafted this article. Yiyu Lai and Juan Kong analyzed the data, reviewed the data, and revised the draft. Chunhong Jia and Zhanyuan Xu participated in design research, collecting and analyzing data. Yaoyong Chen, Qiliang Cui and Fan Wu supervised the elaboration of the research design, data analysis, and reviewed the manuscript. All authors have approved the final version of this article.

\section{References}

1. Sweet DG, Carnielli V, Greisen G, Hallman M, Ozek E, Te Pas A, Plavka R, Roehr CC, Saugstad OD, Simeoni U, et al. European Consensus Guidelines on the Management of Respiratory Distress Syndrome - 
2019 Update. Neonatology. 2019;115(4):432-450.

2. Rojas-Reyes MX, Morley CJ, Soll R. Prophylactic versus selective use of surfactant in preventing morbidity and mortality in preterm infants. Cochrane Database Syst Rev. 2012(3):Cd000510.

3. Zhang L, Cao HY, Zhao S, Yuan LJ, Han D, Jiang H, Wu S, Wu HM. Effect of exogenous pulmonary surfactants on mortality rate in neonatal respiratory distress syndrome: A network meta-analysis of randomized controlled trials. Pulm Pharmacol Ther. 2015;34:46-54.

4. Sardesai S, Biniwale M, Wertheimer F, Garingo A, Ramanathan R. Evolution of surfactant therapy for respiratory distress syndrome: past, present, and future. Pediatr Res. 2017;81(1-2):240-248.

5. Fischer HS, Buhrer C. Avoiding endotracheal ventilation to prevent bronchopulmonary dysplasia: a meta-analysis. Pediatrics.2013;132(5):e1351-1360.

6. Carrasco Loza R, Villamizar Rodriguez G, Medel Fernandez N. Ventilator-Induced Lung Injury (VILI) in Acute Respiratory Distress Syndrome (ARDS): Volutrauma and Molecular Effects. Open Respir Med J. 2015;9:112-119.

7. Leone F, Trevisanuto D, Cavallin F, Parotto M, Zanardo V. Efficacy of INSURE during nasal CPAP in preterm infants with respiratory distress syndrome. Minerva Pediatr. 2013;65(2):187-192.

8. Najafian B, Fakhraie SH, Afjeh SA, Kazemian M, Shohrati M, Saburi A. Early surfactant therapy with nasal continuous positive airway pressure or continued mechanical ventilation in very low birth weight neonates with respiratory distress syndrome. Iran Red Crescent Med J.2014;16(4):e12206.

9. Gomes Cordeiro AM, Fernandes JC, Troster EJ. Possible risk factors associated with moderate or severe airway injuries in children who underwent endotracheal intubation. Pediatr Crit Care Med.2004;5(4):364-368.

10. Klotz D, Porcaro U, Fleck T, Fuchs H. European perspective on less invasive surfactant administration-a survey. Eur J Pediatr.2017;176(2):147-154.

11. Wyckoff M, Aziz K, Escobedo M, et al. Part 13: Neonatal Resuscitation: 2015 American Heart Association Guidelines Update for Cardiopulmonary Resuscitation and Emergency Cardiovascular Care. Circulation. 2015;132:S543-560.

12. Liberati A, Altman DG, Tetzlaff J, Mulrow C, Gøtzsche PC, Ioannidis JP, Clarke M, Devereaux PJ, Kleijnen J, Moher D. The PRISMA statement for reporting systematic reviews and meta-analyses of studies that evaluate health care interventions: explanation and elaboration.PLoS med. 2009;6(7):e1000100.

13. Higgins JP, Green S, editors. Cochrane handbook for systematic reviews of interventions version 5.1.0.. The Cochrane Collaboration. 2011. p 33-49. Available from: www.handbook.cochrane.org.

14. Göpel W, Kribs A, Ziegler A, Laux R, Hoehn T, Wieg C, Siegel J, Avenarius S, von der Wense A, Vochem M, et al. Avoidance of mechanical ventilation by surfactant treatment of spontaneously breathing preterm infants (AMV): an open-label, randomised, controlled trial.Lancet. 2011;378(9803):1627-1634.

15. Heidarzadeh M, Mirnia K, Hoseini MB, Sadeghnia A, Akrami F, Balila M, Ghojazadeh M, Shafai F. Surfactant Administration via Thin Catheter during Spontaneous Breathing: Randomized Controlled Trial in Alzahra hospital. Iran J Neonatol . 2013;4(2):5-9.

16. Kanmaz HG, Erdeve O, Canpolat FE, Mutlu B, Dilmen U. Surfactant administration via thin catheter during spontaneous breathing: randomized controlled trial. Pediatrics. 2013;131(2):e502-509.

17. Mirnia K, Heidarzadeh M, Hosseini MB. Comparison Outcome of Surfactant Administration Via Tracheal Catheterization During Spontaneous Breathing with Insure. Med J Islamic World Acad Sci.2013;21(4):143148.

18. Bao Y, Zhang G, Wu M, Ma L, Zhu J. A pilot study of less invasive surfactant administration in very preterm infants in a Chinese tertiary center. BMC Pediatr. 2015;15:21. 
19. Kribs A, Roll C, Göpel W, Wieg C, Groneck P, Laux R, Teig N, Hoehn T, Böhm W, Welzing L, et al. Nonintubated Surfactant Application vs Conventional Therapy in Extremely Preterm Infants: A Randomized Clinical Trial. JAMA Pediatr. 2015;169(8):723-730.

20. Mohammadizadeh M, Ardestani AG, Sadeghnia AR. Early administration of surfactant via a thin intratracheal catheter in preterm infants with respiratory distress syndrome: Feasibility and outcome. $J$ Res Pharm Pract. 2015;4(1):31-36.

21. Mosayebi Z, Kadivar M, Taheri-Derakhsh N, Nariman S, Mahdi Marashi S, Farsi Z. A Randomized Trial Comparing Surfactant Administration Using InSurE Technique and the Minimally Invasive Surfactant Therapy in Preterm Infants (28 to 34 Weeks of Gestation) with Respiratory Distress Syndrome. J Compr Ped. 2017;In Press(In Press): :e60724.

22. Halim A, Shirazi H, Riaz S, Gul S, Ali W. Less Invasive Surfactant Administration in Preterm Infants with Respiratory Distress Syndrome.J Coll Physicians Surg Pak. 2019;29(3):226-330.

23. Jena SR, Bains HS, Pandita A, et al. Surfactant therapy in premature babies: SurE or InSurE. Pediatr Pulmonol. 2019;54(11):1747-1752.

24. Gupta BK, Saha AK, Mukherjee S, Saha B. Minimally invasive surfactant therapy versus InSurE in preterm neonates of 28 to 34 weeks with respiratory distress syndrome on non-invasive positive pressure ventilation-a randomized controlled trial. Eur J Pediatr.2020;179(8):1287-1293.

25. Han T, Liu H, Zhang H, Guo M, Zhang X, Duan Y, Sun F, Liu X, Zhang X, Zhang M, et al. Minimally Invasive Surfactant Administration for the Treatment of Neonatal Respiratory Distress Syndrome: A Multicenter Randomized Study in China. Front Pediatr. 2020;8:182.

26. Yang G, Hei M, Xue Z, Zhao Y, Zhang X, Wang C. Effects of less invasive surfactant administration (LISA) via a gastric tube on the treatment of respiratory distress syndrome in premature infants aged 32 to 36 weeks. Medicine (Baltimore). 2020;99(9):e19216.

27. Lin HJ, Du LZ, Ma XL, Shi LP, Pan JH, Tong XM, Li QP, Zhou JG, Yi B, Liu L, et al. Mortality and Morbidity of Extremely Low Birth Weight Infants in the Mainland of China: A Multi-center Study. Chin Med J (Engl). 2015;128(20):2743-2750.

28. Wu F, Liu G, Feng Z, Tan X, Yang C, Ye X, Dai Y, Liang W, Ye X, Mo J, et al. Short-term outcomes of extremely preterm infants at discharge: a multicenter study from Guangdong province during 2008-2017. BMC Pediatr. 2019;19(1):405.

29. Clyman RI. The role of patent ductus arteriosus and its treatments in the development of bronchopulmonary dysplasia. Semin Perinatol. 2013;37(2):102-107.

30. Sato K, Nakashima T. Histopathologic changes in laryngeal mucosa of extremely low-birth weight infants after endotracheal intubation. Ann Otol Rhinol Laryngol. 2006;115(11):816-823.

31. Sharma GK, Ahuja GS, Wiedmann M, Osann KE, Su E, Heidari AE, Jing JC, Qu Y, Lazarow F, Wang A, et al. Long-Range Optical Coherence Tomography of the Neonatal Upper Airway for Early Diagnosis of Intubation-related Subglottic Injury. Am J Respir Crit Care Med.2015;192(12):1504-1513.

32. Abdel-Latif M, Osborn D. Nebulised surfactant in preterm infants with or at risk of respiratory distress syndrome. Database Syst Rev. 2012;10:CD008310.

33. Barbosa RF, Simoes ESAC, Silva YP. A randomized controlled trial of the laryngeal mask airway for surfactant administration in neonates.J Pediatr (Rio J). 2017;93(4):343-350.

34. Roberts KD, Brown R, Lampland AL, et al. Laryngeal Mask Airway for Surfactant Administration in Neonates: A Randomized, Controlled Trial.J Pediatr. 2018;193:40-46 e41. 
35. Ten centre trial of artificial surfactant (artificial lung expanding compound) in very premature babies. Ten Centre Study Group. Br Med J (Clin Res Ed). 1987;294(6578):991-996.

36. Barkhuff W, Soll R. Novel Surfactant Administration Techniques: Will They Change Outcome? Neonatology. 2019;115(4):411-422.

37. Kurepa D, Perveen S, Lipener Y, Kakkilaya V. The use of less invasive surfactant administration (LISA) in the United States with review of the literature. J Perinatol. 2019;39(3):426-432.

38. Björklund LJ, Ingimarsson J, Curstedt T, John J, Robertson B, Werner O, Vilstrup CT. Manual ventilation with a few large breaths at birth compromises the therapeutic effect of subsequent surfactant replacement in immature lambs. Pediatr Res. 1997;42(3):348-355.

39. Herting E, Hartel C, Gopel W. Less invasive surfactant administration (LISA): chances and limitations. Arch Dis Child Fetal Neonatal Ed. 2019;104(6):F655-F659.

40. More K, Sakhuja P, Shah PS. Minimally invasive surfactant administration in preterm infants: a metanarrative review. JAMA Pediatr. 2014;168(10):901-908.

41. Isayama T, Iwami H, McDonald S, Beyene J. Association of Noninvasive Ventilation Strategies With Mortality and Bronchopulmonary Dysplasia Among Preterm Infants: A Systematic Review and Metaanalysis. JAMA. 2016;316(6):611-624.

42. Langhammer K, Roth B, Kribs A, Göpel W, Kuntz L, Miedaner F. Treatment and outcome data of very low birth weight infants treated with less invasive surfactant administration in comparison to intubation and mechanical ventilation in the clinical setting of a cross-sectional observational multicenter study. Eur $J$ Pediatr.2018;177(8):1207-1217.

43. Aldana-Aguirre JC, Pinto M, Featherstone RM, Kumar M. Less invasive surfactant administration versus intubation for surfactant delivery in preterm infants with respiratory distress syndrome: a systematic review and meta-analysis. Arch Dis Child Fetal Neonatal Ed.2017;102(1):F17-F23.

\section{Hosted file}

Table.pdf available at https://authorea.com/users/395138/articles/508463-efficacy-andsafety-of-minimally-invasive-surfactant-administration-in-preterm-infants-with-neonatalrespiratory-distress-syndrome-a-systematic-review-and-meta-analysis 


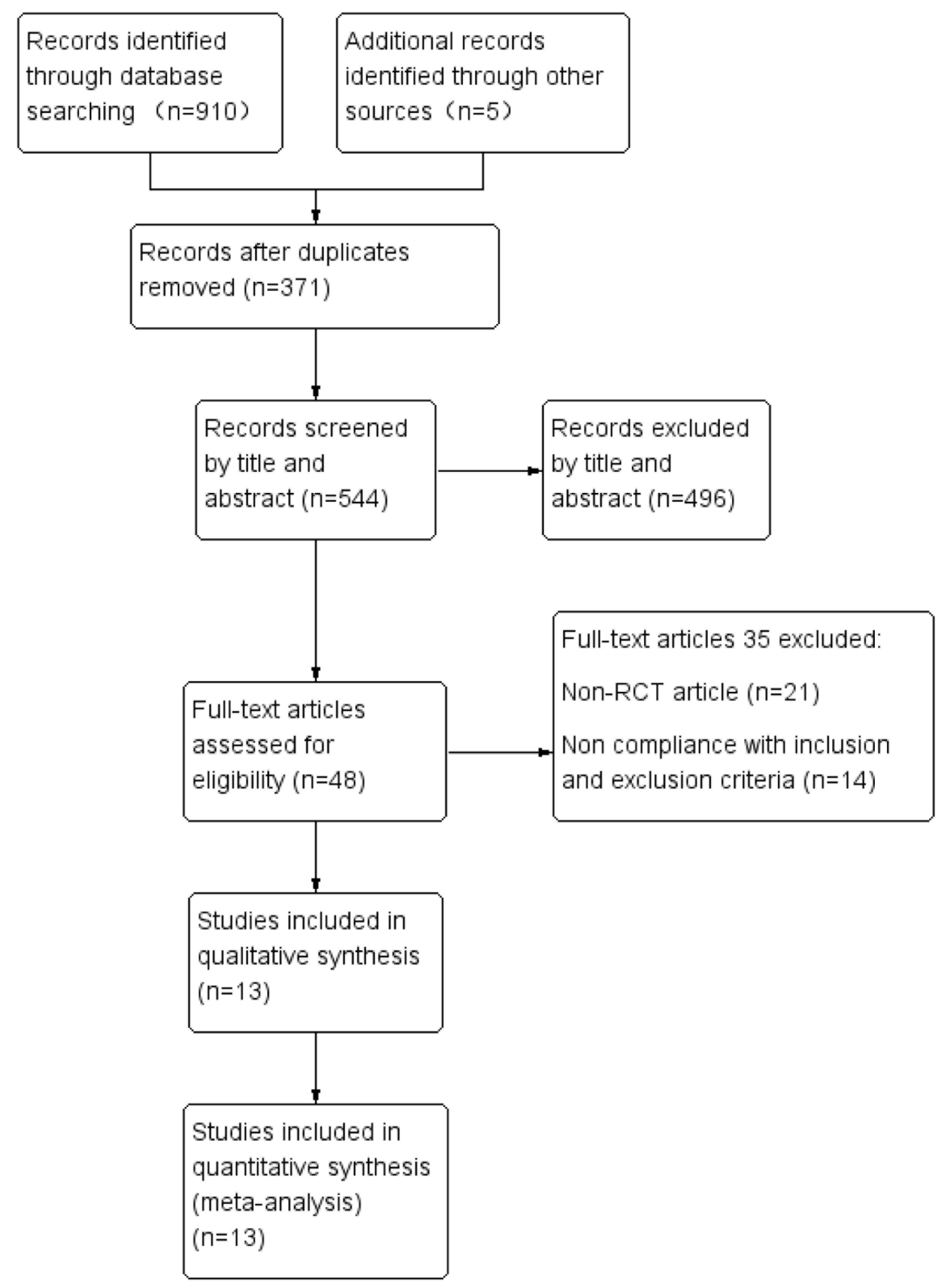




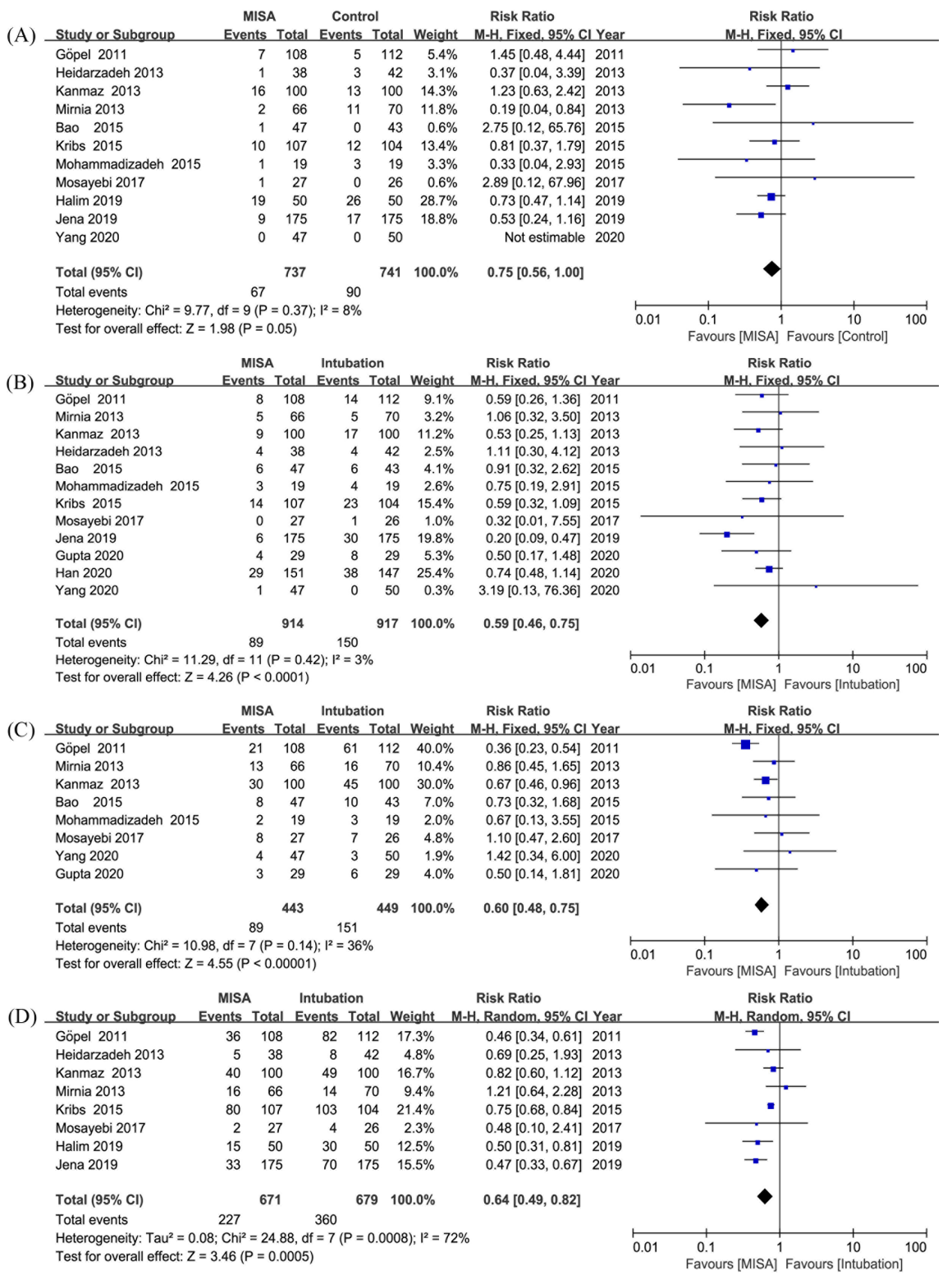




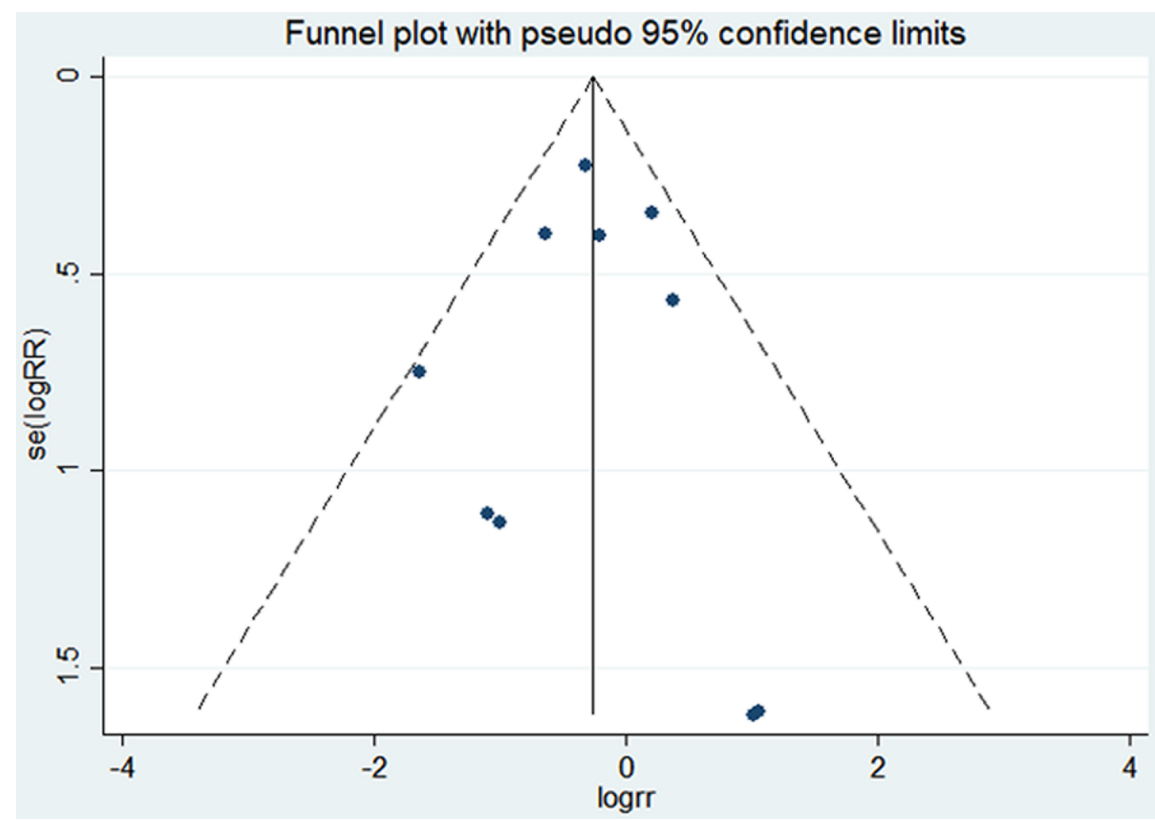

